\title{
Oscillations for Certain Difference Equations with Continuous Variable
}

\author{
Binggen Zhang and Linlin Zhao
}

\begin{abstract}
In this paper, we investigate some nonlinear difference equations with continuous variable. A linearized oscillation result is established and oscillation criteria for some forced difference equations are obtained.
\end{abstract}

Keywords: Difference equations, positive solutions, oscillation

AMS subject classification: $39 \mathrm{~A}$

\section{Introduction}

Recently, there has been an increasing interest in the study of the oscillatory behavior of the solutions of delay difference equations [1]. In [3], authors consider the oscillation of the delay difference equation

$$
y(t)-y(t-\tau)+p(t) H(y(t-\sigma))=f(t) \quad(t \geq 0)
$$

where $\tau, \sigma>0$, and $p \in C\left(\mathbb{R}_{+}, \mathbb{R}_{+}\right), f \in C\left(\mathbb{R}_{+}, \mathbb{R}\right)$ and $H \in C(\mathbb{R}, \mathbb{R})$. In the present paper we use some ideas from [3] to consider the oscillation of the equation

$$
y(t-\tau)-y(t)+\sum_{i=1}^{m} p_{i} f_{i}\left(y\left(t+\sigma_{i}\right)\right)=0
$$

where $\tau>0, \sigma_{m} \geq \ldots \geq \sigma_{1}>0, p_{i}>0, f_{i} \in C(\mathbb{R}, \mathbb{R}), u f_{i}(u)>0$ for $u \neq 0$ and $\lim _{u \rightarrow \infty} \frac{f_{i}(u)}{u}=1$, and of the equation

$$
y(t)-a(t) y(t-\tau)+G(t, y(t-\sigma))=f(t)
$$

where $\tau, \sigma>0$ and $a \in C\left(\mathbb{R}_{+}, \mathbb{R}_{+}\right), G \in C\left(R_{+} \times \mathbb{R}, \mathbb{R}\right)$ and $f \in C\left(\mathbb{R}_{+}, \mathbb{R}\right)$. As usuall, a solution of equation (1) or (2) is said to be oscillatory if it is neither eventually positive nor eventually negative. Otherwise the solution is called non-oscillatory. In Section 1, we obtain a linearized oscillation result for equation (1) and in Section 2 we obtain some oscillation criteria for the forced equation (2).

Both authors: Ocean Univ. of Qingdao, Dept. Math., Qingdao 266003, China bgzhang@public.qd.sd.cn. This work is supported by NNSF of China 


\section{Linearized oscillation for equation (1)}

Consider equation (1) together with the associated linear difference equation

$$
y(t-\tau)-y(t)+\sum_{i=1}^{m} p_{i} y\left(t+\sigma_{i}\right)=0 .
$$

The first lemma is borrowed from [2].

Lemma 1. The following statements are equivalent:

(a) Every solution of equation (3) oscillates.

(b) The characteristic equation

$$
e^{-\lambda \tau}-1+\sum_{i=1}^{m} p_{i} e^{\lambda \sigma_{i}}=0
$$

has no real roots.

Lemma 2. Every solution of equation (3) oscillates if and only if the inequality

$$
y(t-\tau)-y(t)+\sum_{i=1}^{m} p_{i} y\left(t+\sigma_{i}\right) \leq 0
$$

has no eventually positive solutions.

Proof. Necessity. Suppose $y>0$ is an eventually positive solution of equation (5). Then

$$
y(t) \geq y(t-\tau)+\sum_{i=1}^{m} p_{i} y\left(t+\sigma_{i}\right) \quad(t \geq T-\tau>0)
$$

The further proof is simple and based on the following Knaster Fixed Point Theorem [4]:

Let $(X, \leq)$ be an ordered set, let for every subset $M$ of $X$ there exist inf $M$ and $\sup M$, and let $T: M \rightarrow M$ be an increasing mapping, that is, $x \leq y$ implies $T x \leq T y$. Then there exists at least one element $x \in X$ such that $T x=x$.

To use this theorem, define the set

$$
X=\{x \in C: 0 \leq x(t) \leq y(t) \quad(t \geq T-\tau)\}
$$


endowed with usual pointwise ordering, i.e. $x_{1} \leq x_{2}$ if $x_{1}(t) \leq x_{2}(t)$ for every $t \geq T-\tau$. It is easy to see that every $A \subseteq X$ has a supremum which belongs to $X$. Define an operator $S$ on $X$ by

$$
(S x)(t)= \begin{cases}x(t-\tau)+\sum_{i=1}^{m} p_{i} x\left(t+\sigma_{i}\right) & \text { if } t \geq T \\ \left(1-\frac{t}{T}\right) y(t)+\frac{t y(t) S x(T)}{T y(T)} & \text { if } T-\tau \leq t<T .\end{cases}
$$

For any $x \in X$, from

$$
0 \leq(S x)(t)=x(t-\tau)+\sum_{i=1}^{m} p_{i} x\left(t+\sigma_{i}\right) \leq y(t-\tau)+\sum_{i=1}^{m} p_{i} y\left(t+\sigma_{i}\right) \leq y(t)
$$

for $t \geq T$ and

$$
0 \leq(S x)(t)=\left(1-\frac{t}{T}\right) y(t)+\frac{t y(t) S x(T)}{T y(T)} \leq\left(1-\frac{t}{T}\right) y(t)+\frac{t}{T} y(t)=y(t)
$$

for $T-\tau \leq t<T$ we know that $S X \subseteq X$. Moreover, $S$ is obviously nondecreasing. By the Knaster Fixed Point Theorem, there exists an $x^{*} \in X$ such that $S x^{*}=x^{*}$. As $T \leq t \leq T+\tau$,

$$
\begin{aligned}
x^{*}(t) & =x^{*}(t-\tau)+\sum_{i=1}^{m} p_{i} x^{*}\left(t+\sigma_{i}\right) \\
& \geq x^{*}(t-\tau) \\
& =S x^{*}(t-\tau) \\
& =\left(1-\frac{t-\tau}{T}\right) y(t-\tau)+\frac{(t-\tau) y(t-\tau) S x^{*}(T)}{T y(T)} \\
& \geq\left(1-\frac{t-\tau}{T}\right) y(t-\tau) \\
& >0
\end{aligned}
$$

Repeating this procedure, we get $x^{*}(t)>0$ as $t \geq T$. So $x^{*}$ is an eventually positive solution of equation (3), which is a contradiction.

Sufficiency. Suppose equation (3) has an eventually positive solution $y>0$ or eventually negative solution $x<0$. Because the latter means $-x>0$ is an eventually positive solution of equation (3), we only discuss the former case. It is easy to show that equation (5) has an eventually positive solution $y>0$. This is a contradiction 
Lemma 3. Assume that $u f_{i}(u)>0$ for $u \neq 0, \lim _{u \rightarrow \infty} \frac{f_{i}(u)}{u}=1$ and $f_{i}$ is convex for $u>0$ and concave for $u<0 \quad(i=1,2, \ldots, m)$. Further, assume that every solution of the equation

$$
y(t-\tau)-y(t)+(1-\varepsilon) \sum_{i=1}^{m} p_{i} y\left(t+\sigma_{i}\right)=0 \quad(\varepsilon \in(0,1))
$$

oscillates. Then every solution of equation (1) oscillates.

Proof. Suppose equation (1) has an eventually positive solution $y$ and set $z(t)=\frac{1}{\tau} \int_{t-\tau}^{t} y(s) d s>0$. Then

$$
z^{\prime}(t)=\frac{1}{\tau}(y(t)-y(t-\tau))=\frac{1}{\tau} \sum_{i=1}^{m} p_{i} f_{i}\left(y\left(t+\sigma_{i}\right)\right)>0
$$

eventually. Hence $\lim _{t \rightarrow \infty} z(t)=\beta>0$ exists. We claim that $\beta=\infty$. Otherwise, $0<\beta<\infty$. We integrate equation (1) from $t-\tau$ to $t$ and get

$$
\int_{t-\tau}^{t} y(s-\tau) d s-\int_{t-\tau}^{t} y(s) d s+\sum_{i=1}^{m} p_{i} \int_{t-\tau}^{t} f_{i}\left(y\left(s+\sigma_{i}\right)\right) d s=0
$$

Since $f_{i}$ is convex for $u>0$, by Jensen's inequality we have

$$
z(t-\tau)-z(t)+\sum_{i=1}^{m} p_{i} f_{i}\left(z\left(t+\sigma_{i}\right)\right) \leq 0
$$

Letting $t \rightarrow \infty$, from (10) we obtain the inequality $\sum_{i=1}^{m} p_{i} f_{i}(\beta) \leq 0$ which is a contradiction. By $\lim _{u \rightarrow \infty} \frac{f_{i}(u)}{u}=1$ for any $\varepsilon \in(0,1)$ there exists $\alpha>0$ such that

$$
(1-\varepsilon) u<f_{i}(u)<(1+\varepsilon) u \quad(u \geq \alpha)
$$

Thus, from (10) we have

$$
z(t-\tau)-z(t)+(1-\varepsilon) \sum_{i=1}^{m} p_{i} z\left(t+\sigma_{i}\right) \leq 0
$$

By Lemma 2, equation (8) has a positive solution. This is a contradiction. Similarly, we can prove that equation (1) have no eventually negative solutions 


\section{Lemma 4. If}

$$
y(t-\tau)-y(t)+(1+\varepsilon) \sum_{i=1}^{m} p_{i} y\left(t+\sigma_{i}\right)=0 \quad(\varepsilon \in(0,1))
$$

has positive solutions and $f_{i}$ is non-decreasing in $u$, so does equation (1).

Proof. By Lemma 1 and the fact that equation (12) has an eventually positive solution, the characteristic equation

$$
e^{-\lambda \tau}-1+(1+\varepsilon) \sum_{i=1}^{m} p_{i} e^{\lambda \sigma_{i}}=0
$$

has a real root $\eta$. Clearly, $\eta>0$. Thus $e^{\eta t}$ is a solution of equation (12) which tends to infinity as $t \rightarrow \infty$. Suppose $y(t) \rightarrow \infty$ as $t \rightarrow \infty$ is a positive solution of equation (12). From (11) we have

$y(t-\tau)-y(t)+\sum_{i=1}^{m} p_{i} f_{i}\left(y\left(t+\sigma_{i}\right)\right) \leq y(t-\tau)-y(t)+(1+\varepsilon) \sum_{i=1}^{m} p_{i} y\left(t+\sigma_{i}\right)=0$.

Then

$$
y(t) \geq y(t-\tau)+\sum_{i=1}^{m} p_{i} f_{i}\left(y\left(t+\sigma_{i}\right)\right)
$$

Define

$$
Y=\{a \in C: 0 \leq a(t) \leq y(t) \text { for } t \geq T-\tau\}
$$

and an operator $E$ on $Y$ by

$$
E a(t)= \begin{cases}a(t-\tau)+\sum_{i=1}^{m} p_{i} f_{i}\left(a\left(t+\sigma_{i}\right)\right) & \text { if } t \geq T \\ \left(1-\frac{t}{T}\right) y(t)+\frac{\operatorname{ty}(t) \operatorname{Ex}(T)}{\operatorname{Ty}(T)} & \text { if } T-\tau \leq t<T .\end{cases}
$$

Similar to the proof of Lemma 2, we can prove that there exists a fixed point $a \in Y$ and $a(t)>0$ for $t \geq T$. Since $a=E a, a$ is a positive solution of equation (1)

Lemma 5. The equation

$$
F(\lambda)=e^{-\lambda \tau}-1+\sum_{i=1}^{m} p_{i} e^{\lambda \sigma_{i}}=0
$$

has real roots if and only if there exists $\varepsilon_{0} \in(0,1)$ such that

$$
e^{-\lambda \tau}-1+(1+\varepsilon) \sum_{i=1}^{m} p_{i} e^{\lambda \sigma_{i}}=0 \quad\left(|\varepsilon|<\varepsilon_{0}\right)
$$


has real roots.

Proof. Sufficiency. If there exists $\varepsilon_{0} \in(0,1)$ such that equation (16) has real roots, then let $\varepsilon=0$ and we obtain that the equation

$$
F(\lambda)=e^{-\lambda \tau}-1+\sum_{i=1}^{m} p_{i} e^{\lambda \sigma_{i}}=0
$$

has real roots.

Necessity. Suppose $F(\lambda)=0$ has a real root $\eta$, i.e. $F(\eta)=0$. Define a function $H$ as

$$
H(\varepsilon, \lambda)=e^{-\lambda \tau}-1+(1+\varepsilon) \sum_{i=1}^{m} p_{i} e^{\lambda \sigma_{i}} \quad(|\varepsilon|<1) .
$$

It is easy to see that $H \in C((-1,1) \times \mathbb{R}, \mathbb{R})$ and

$$
H(0, \eta)=e^{-\eta \tau}-1+\sum_{i=1}^{m} p_{i} e^{\eta \sigma_{i}}=F(\eta)=0 .
$$

In a small neighbourhood of $(0, \eta)$ the equation $H(\varepsilon, \lambda(\varepsilon))=0$ defines a continuous function $\lambda=\lambda(\varepsilon)$ which satisfies $H(\varepsilon, \lambda(\varepsilon))=0, \lambda(0)=\eta$ and $\lim _{\varepsilon \rightarrow 0} \lambda(\varepsilon)=\eta$. So there exists $\varepsilon_{0} \in(0,1)$ such that equation (16) has real roots

From the above lemmas, we can describe the first main result in this paper.

Theorem 1. Assume that $p_{i}, \tau, \sigma_{i}>0$ and $f_{i} \in C(\mathbb{R}, \mathbb{R}), u f_{i}(u)>0$ for $u \neq 0, \lim _{u \rightarrow \infty} \frac{f_{i}(u)}{u}=1, f_{i}$ is non-decreasing in $u$, convex for $u>0$ and concave for $u<0 \quad(i=1,2, \ldots, m)$. Then every solution of equation (1) oscillates if and only if every solution of equation (3) oscillates.

Proof. Sufficiency. If the solution of equation (3) oscillates, by Lemma 1 equation (4) and hence by Lemma 5 equation (16) has no real roots. By Lemma 1, every solution of equation (8) oscillates. From Lemma 3 we show that every solution of equation (1) oscillates.

Necessity. Suppose equation (3) has an eventually positive solution. By Lemma 1 equation (4) and by Lemma 5 equation (16) has real roots. By Lemma 1

$$
y(t-\tau)-y(t)+(1+\varepsilon) \sum_{i=1}^{m} p_{i} y\left(t+\sigma_{i}\right)=0 \quad\left(|\varepsilon|<\left|\varepsilon_{0}\right|\right)
$$

and by Lemma 4 equation (1) has eventually positive solutions, which is a contradiction 


\section{Oscillations for equation (2)}

The following lemma will be used to state the main results in Section 2 .

Lemma 6. Assume that $f \in C\left(\mathbb{R}_{+}, \mathbb{R}\right)$ and $a(t) \neq 0$ as $t \geq T$ where $T \geq \tau$. Then there exists a continuous function $F=F(t)$ as $t \geq T-\tau$ such that $F(t)-F(t-\tau) a(t)=f(t)$ for $t \geq T$.

Proof. Define

$$
a_{1}(t)= \begin{cases}a(t) & \text { if } t \geq T \\ \frac{t-T+\tau}{\tau} a_{1}(T) & \text { if } T-\tau \leq t<T \\ 0 & \text { if } t<T-\tau .\end{cases}
$$

Then $a_{1} \in C(\mathbb{R}, \mathbb{R})$ and $a_{1}(t)=a(t)$ for $t \geq T$. Define

$$
r(t)= \begin{cases}f(t) a^{-1}(t) & \text { if } t \geq T \\ \frac{t-T+\tau}{\tau} r(T) & \text { if } T-\tau \leq t<T \\ 0 & \text { if } t<T-\tau .\end{cases}
$$

Then $r \in C(\mathbb{R}, \mathbb{R})$. Let

$$
F(t)=\sum_{i=0}^{\infty} r(t-i \tau) \prod_{j=0}^{i} a_{1}(t-j \tau) \quad(t \geq T) .
$$

Obviously, $F \in C\left(\mathbb{R}_{+}, \mathbb{R}\right)$. When $t \geq T$, we know

$$
\begin{aligned}
F(t) & -a(t) F(t-\tau) \\
& =\sum_{i=0}^{\infty} r(t-i \tau) \prod_{j=0}^{i} a_{1}(t-j \tau)-a(t) \sum_{i=0}^{\infty} r(t-\tau-i \tau) \prod_{j=0}^{i} a_{1}(t-\tau-j \tau) \\
& =\sum_{i=0}^{\infty} r(t-i \tau) \prod_{j=0}^{i} a_{1}(t-j \tau)-\sum_{i=1}^{\infty} r(t-i \tau) \prod_{j=0}^{i} a_{1}(t-j \tau) \\
& =r(t) a(t) \\
& =f(t)
\end{aligned}
$$

and the proof is complete

Set

$$
\begin{array}{r}
\bar{y}(t)=\int_{T^{\prime}}^{t} y(s) d s+\int_{t-\tau}^{T^{\prime}} a(s+\tau) y(s) d s \\
\bar{F}(t)=\int_{T^{\prime}}^{t} F(s) d s+\int_{t-\tau}^{T^{\prime}} a(s+\tau) F(s) d s
\end{array}
$$

where

$$
T^{\prime}=\left\{\begin{array}{ll}
t-\frac{\tau}{2} & \text { if } a(s+\tau) \in(0,1] \\
t-\frac{3 \tau}{2} & \text { if } a(s+\tau) \in(1,+\infty)
\end{array} \quad(s \in[t-\tau, t])\right.
$$

and set $\bar{F}_{ \pm}(t)=\max \{ \pm \bar{F}(t), 0\}$. 
Theorem 2. Assume the following:

(a) $g(t, u)=\min _{t-\tau \leq s \leq t} G(s, u)$ for $u>0$.

(b) $G(t, u)$ is an odd function in $u, u G(t, u)>0$ for $u \neq 0, g(t, 0)=0$, and $g(t, u)$ is non-decreasing and is convex in $u>0$.

(c) For any number $N>0$, there exist two sequences $\left\{t_{i}\right\}$ and $\left\{t_{i}^{\prime}\right\}$ such that $t_{i+1}-t_{i} \geq \tau$ and $t_{i+1}^{\prime}-t_{i}^{\prime} \geq \tau$, and $a(t) \in(0,1]$ or $a(t) \in(1,+\infty)$ as $t \in\left[t_{i}-\sigma-2 \tau, t_{i}-\sigma-\tau\right] \quad(i=1,2, \ldots)$, and

$$
\begin{aligned}
& \sum_{i=1}^{\infty} \tau g\left(t_{i}, \frac{1}{\tau} \bar{F}_{+}\left(t_{i}-\sigma\right)\right)>N \\
& \sum_{i=1}^{\infty} \tau g\left(t_{i}^{\prime}, \frac{1}{\tau} \bar{F}_{-}\left(t_{i}^{\prime}-\sigma\right)\right)>N .
\end{aligned}
$$

Then every solution of equation (2) oscillates.

Proof. From Lemma 6, equation (2) can be rewritten in the form

$$
(y(t)-F(t))-a(t)(y(t-\tau)-F(t-\tau))+G(t, y(t-\sigma))=0 .
$$

Suppose the contrary, let $y>0$ be an eventually positive solution of equation (19) and let $z=\bar{y}-\bar{F}$. Then equation (19) becomes

$$
z^{\prime}(t)+G(t, y(t-\sigma))=0 .
$$

So $z^{\prime}(t)<0$ for $t \geq T$. If $z(t)<0$ eventually, then $0<\bar{y}(t)<\bar{F}(t)$ eventually and hence $\bar{F}_{-}(t)=0$ and $g\left(t, \frac{1}{\tau} \bar{F}_{-}(t-\sigma)\right)=0$ which contradicts (18). Therefore $z(t)>0$ and $\lim _{t \rightarrow \infty} z(t)=\alpha \geq 0$ exists. Integrating equation (20) from $T$ to $\infty$ we obtain

$$
\int_{T}^{\infty} G(t, y(t-\sigma)) d t=z(T)-\alpha<\infty .
$$

Since $z(t)>0$, we have $\bar{y}(t) \geq \bar{F}(t)$ and hence $\bar{y}(t) \geq \bar{F}_{+}(t)$ for $t \geq T$. There exists $k>0$ such that $t_{k}-\tau \geq T+\sigma$ and so by Jensen's inequality

$$
\begin{aligned}
\int_{T+\sigma}^{\infty} G(t, y(t-\sigma)) d t & \geq \sum_{i=k}^{\infty} \int_{t_{i}-\tau}^{t_{i}} G(t, y(t-\sigma)) d t \\
& \geq \sum_{i=k}^{\infty} \int_{t_{i}-\tau}^{t_{i}} g(t, y(t-\sigma)) d t \\
& \geq \sum_{i=k}^{\infty} \tau g\left(t_{i}, \frac{1}{\tau} \int_{t_{i}-\tau}^{t_{i}} y(t-\sigma) d t\right) .
\end{aligned}
$$


Setting $A_{i}=\left[t_{i}-2 \tau-\sigma, t_{i}-\tau-\sigma\right]$ we obtain

$$
\begin{aligned}
& \int_{t_{i}-\tau}^{t_{i}} y(s-\sigma) d s \\
& =\left\{\begin{array}{l}
\int_{t_{i}-\frac{\tau}{2}}^{t_{i}} y(s-\sigma) d s+\int_{t_{i}-\tau}^{t_{i}-\frac{\tau}{2}} y(s-\sigma) d s \quad \text { if } a \in C\left(A_{i},(0,1]\right) \\
\int_{t_{i}-\frac{3 \tau}{2}}^{t_{2}} y(s-\sigma) d s+\int_{t_{i}-\tau}^{t_{i}-\frac{3 \tau}{2}} y(s-\sigma) d s \quad \text { if } a \in C\left(A_{i},(1,+\infty)\right)
\end{array}\right. \\
& \geq\left\{\begin{array}{l}
\int_{t_{i}-\frac{\tau}{2}-\sigma}^{t_{i}-\sigma} y(s) d s+\int_{t_{i}-\tau-\sigma}^{t_{i}-\frac{\tau}{2}-\sigma} a(s+\tau) y(s) d s \quad \text { if } a \in C\left(A_{i},(0,1]\right) \\
\int_{t_{i}-\frac{3 \tau}{2}-\sigma}^{t_{i}} y(s) d s-\int_{t_{i}-\frac{3 \tau}{2}-\sigma}^{t_{i}-\tau} a(s+\tau) y(s) d s \quad \text { if } a \in C\left(A_{i},(1,+\infty)\right) \\
=\bar{y}\left(t_{i}-\sigma\right) \\
\geq \bar{F}_{+}\left(t_{i}-\sigma\right) .
\end{array}\right.
\end{aligned}
$$

In view of $(21)-(23)$ and that $g(t, u)$ is non-decreasing in $u>0$ we have

$$
z(T)>\int_{T+\sigma}^{\infty} G(t, y(t-\sigma)) d t \geq \sum_{i=k}^{\infty} \tau g\left(t_{i}, \frac{1}{\tau} \bar{F}_{+}\left(t_{i}-\sigma\right)\right)
$$

which contradicts (17). Suppose $y<0$ is an eventually negative solution of equation (19). Then similarly we can prove that $z^{\prime}>0, z<0$, hence $\lim _{t \rightarrow \infty} z(t)=\beta \leq 0$ and

$$
\begin{aligned}
\infty & >\beta-z(T) \\
& =-\int_{T}^{\infty} G(t, y(t-\sigma)) d t \\
& >-\int_{T+\sigma}^{\infty} G(t, y(t-\sigma)) d t \\
& \geq \sum_{i=k}^{\infty}\left(-\int_{t_{i}^{\prime}-\tau}^{t_{i}^{\prime}} G(t, y(t-\sigma)) d t\right) \\
& =\sum_{i=k}^{\infty} \int_{t_{i}^{\prime}-\tau}^{t_{i}^{\prime}} G(t,-y(t-\sigma)) d t \\
& \geq \sum_{i=k}^{\infty} \int_{t_{i}^{\prime}-\tau}^{t_{i}^{\prime}} g\left(t_{i}^{\prime},-y(t-\sigma)\right) d t \\
& \geq \sum_{i=k}^{\infty} \tau g\left(t_{i}^{\prime},-\frac{1}{\tau} \bar{y}\left(t_{i}^{\prime}-\sigma\right)\right) .
\end{aligned}
$$

Since $z<0, \bar{y}<\bar{F}$ and hence $-\bar{y}>-\bar{F}$, therefore $-\bar{y}(t)>(-\bar{F}(t))_{+}=\bar{F}{ }_{-}(t)$. From (24) we have

$$
\sum_{i=k}^{\infty} \tau g\left(t_{i}^{\prime}, \frac{1}{\tau} \bar{F}_{-}\left(t_{i}^{\prime}-\sigma\right)\right)<-z(T)
$$


which contradicts (18)

Example 1. Consider the difference equation

$$
y(t)-t y(t-\pi)+(1+t) y^{3}\left(t-\frac{\pi}{2}\right)=(1+t)\left(\cos t+\sin ^{3} t\right) .
$$

In this case $G(t, u)=(1+t) u^{3}, f(t)=(1+t)\left(\cos t+\sin ^{3} t\right), \sigma=\frac{\pi}{2}, \tau=\pi$, $a(t)=t$ and $F(t)=\cos t+\sin ^{3} t$. Let $T=\frac{5 \pi}{2}+1$. So $a(t)>1$ as $t \geq T$. Thus

$$
\begin{aligned}
\bar{F}(t) & =\int_{t-\frac{3 \pi}{2}}^{t}\left(\cos s+\sin ^{3} s\right) d s+\int_{t-\pi}^{t-\frac{3 \pi}{2}}(s+\pi)\left(\cos s+\sin ^{3} s\right) d s \\
& =\left(\frac{4}{9}+\frac{\pi}{6}-\frac{t}{3}\right) \sin ^{3} t+\left(\frac{4}{9}+\frac{t}{3}\right) \cos ^{3} t+\left(2 t-\frac{\pi}{2}-\frac{1}{3}\right) \sin t-\left(\frac{\pi}{2}+\frac{1}{3}\right) \cos t \\
g(t, u) & =\min _{t-\pi \leq s \leq t}\left\{(1+s) u^{3}\right\}=(1+t-\pi) u^{3} \quad(u>0) .
\end{aligned}
$$

It is easy to see that the former two coditions of Theorem 2 hold. We only need to show that (17) and (18) could be fulfilled. In fact, let $t_{1}=3 \pi, t_{n}=t_{n-1}+2 \pi$ and $t_{1}^{\prime}=\frac{9 \pi}{2}, t_{n}^{\prime}=t_{n-1}^{\prime}+2 \pi$, i.e. two sequences $\left\{t_{i}\right\},\left\{t_{i}^{\prime}\right\} \quad(i \geq 1)$ exist and

$$
\begin{aligned}
& \bar{F}_{+}\left(t_{i}-\frac{\pi}{2}\right)=\frac{5}{3} t_{i}+\frac{1}{9}-\frac{7}{6} \pi \geq 5 \pi+\frac{1}{9}-\frac{7}{6} \pi>3 \pi \\
& \sum_{i=1}^{\infty} \pi g\left(t_{i}, \frac{1}{\pi} \bar{F}_{+}\left(t_{i}-\sigma\right)\right)=\frac{1}{\pi^{2}} \sum_{i=1}^{\infty}\left(1+t_{i}-\pi\right)\left(\bar{F}_{+}\left(t_{i}-\sigma\right)\right)^{3} .
\end{aligned}
$$

In view of $\left(1+t_{i}-\pi\right)\left(\bar{F}_{+}\left(t_{i}-\sigma\right)\right)^{3}>54 \pi^{4}, \sum_{i=1}^{\infty} \pi g\left(t_{i}, \frac{1}{\pi} \bar{F}_{+}\left(t_{i}-\sigma\right)\right)=\infty$. Analogously, $\bar{F}_{-}\left(t_{i}^{\prime}-\frac{\pi}{2}\right)=\frac{t_{i}^{\prime}}{3}-\frac{2}{3} \pi+\frac{1}{9}>\frac{5}{6} \pi$ and so $\sum_{i=1}^{\infty} \pi g\left(t_{i}^{\prime}, \frac{1}{\pi} \bar{F}_{-}\left(t_{i}^{\prime}-\frac{\pi}{2}\right)\right)=$ $\infty$. Therefore (17) and (18) are satisfied. By Theorem 2, the solutions of equation (25) oscillate. Actually, $y=\cos t$ is a such solution of equation (25).

If $a(t)=1$ and $G(t, y(t-\sigma))=p(t) y(t-\sigma)$, then equation (2) becomes

$$
y(t)-y(t-\tau)+p(t) y(t-\sigma)=f(t) .
$$

Corollary 1. Suppose $p \in C\left(\mathbb{R}_{+}, \mathbb{R}_{+}\right)$and for any number $N>0$ there exist two sequences $\left\{t_{i}\right\}$ and $\left\{t_{i}^{\prime}\right\}$ such that $t_{i+1}-t_{i}, t_{i+1}^{\prime}-t_{i}^{\prime} \geq \tau(i \geq 1)$ and

$$
\sum_{i=1}^{\infty} q\left(t_{i}\right) \bar{F}_{+}\left(t_{i}-\sigma\right)>N \quad \text { and } \quad \sum_{i=1}^{\infty} q\left(t_{i}^{\prime}\right) \bar{F}_{-}\left(t_{i}^{\prime}-\sigma\right)>N
$$

where $q(t)=\min _{t-\tau \leq s \leq t} p(s)$. Then every solution of equation (26) oscillates.

Corollary 1 is [3: Theorem 2.5]. Similar to [3], from Theorem 2 we can obtain the following conclusion. 
Corollary 2. Assume conditions (a) - (b) in Theorem 2 and either $a(t) \in$ $(0,1]$ or $a(t) \in(1,+\infty)$ as $t \geq T$. Furthermore, let $\int_{T}^{\infty} g\left(t, \frac{1}{\tau} \bar{F}_{ \pm}(t-\sigma)\right) d t=$ $\infty$. Then every solution of equation (2) oscillates.

By the bivariate Jensen inequality we can get the next oscillation criterion.

Theorem 3. Assume the following:

(i) $G(t, u)$ is non-decreasing and is an odd function in $u, G(t, 0)=0$, $u G(t, u)>0$ for $u \neq 0$ and $G(t, u)$ is convex in $(t, u)$ as $t, u>0$.

(ii) Condition (c) of Theorem 2 holds where (17) and (18) are replaced by

$\sum_{i=1}^{\infty} \tau G\left(t_{i}-\frac{\tau}{2}, \frac{1}{\tau} \bar{F}_{+}\left(t_{i}-\sigma\right)\right)>N \quad$ and $\quad \sum_{i=1}^{\infty} \tau G\left(t_{i}^{\prime}-\frac{\tau}{2}, \frac{1}{\tau} \bar{F}_{-}\left(t_{i}^{\prime}-\sigma\right)\right)>N$

respectively. Then every solution of equation (2) oscillates.

The proof of Theorem 3 is similar to that of Theorem 2 . We only need to pay attention to Jensen's inequality for functions in two variables, i.e.

$$
\frac{1}{\tau} \int_{t-\tau}^{t} G(s, y(s)) d s \geq G\left(t-\frac{\tau}{2}, \frac{1}{\tau} \int_{t-\tau}^{t} y(s) d s\right)
$$

since $G(t, u)$ is convex in $(t, u)$.

Similar to Corollary 1 , for the linear equation (26) we have

Corollary 3. Assume $p \in C\left(\mathbb{R}_{+}, \mathbb{R}_{+}\right)$and up $(t)$ is convex in $(t, u)$ as $t, u>0$. Furthermore, for any number $N>0$ let there exist two sequences $\left\{t_{i}\right\}$ and $\left\{t_{i}^{\prime}\right\}$ such that $t_{i+1}-t_{i}, t_{i+1}^{\prime}-t_{i}^{\prime} \geq \tau \quad(i \geq 1)$,

$$
\sum_{i=1}^{\infty} p\left(t_{i}-\frac{\tau}{2}\right) \bar{F}_{+}\left(t_{i}-\sigma\right)>N \quad \text { and } \quad \sum_{i=1}^{\infty} p\left(t_{i}^{\prime}-\frac{\tau}{2}\right) \bar{F}_{-}\left(t_{i}^{\prime}-\sigma\right)>N
$$

Then every solution of equation (26) oscillates.

Corollary 4. Assume condition (i) of Theorem 3 holds, either a $(t) \in$ $(0,1]$ or $a(t) \in(1,+\infty)$ as $t \geq T$, and $\int_{T}^{\infty} G\left(t-\frac{\tau}{2}, \frac{1}{\tau} \bar{F}_{ \pm}(t-\sigma)\right) d t=\infty$. Then every solution of equation (2) oscillates.

We can also extend the above methods to investigate the oscillation of the solution of the difference equation

$$
a(t) y(t-\tau)-y(t)+G(t, y(t+\sigma))=f(t)
$$




\section{References}

[1] Agarwal, R. P.: Difference Equations and Inequalities. New York: Marcel Dekker 2000.

[2] Ladas, G., Pakala, L. and Z. Wang: Necessary and sufficient conditions for the oscillation of difference equations. PanAmer. Math. J. 2 (1992), $17-26$.

[3] Zhang, B. G., Jun Ni and Sung Kyu Choi: Oscillation for difference equations with continuous variable. Comp. Math. Appl. 36 (1998)9, 11 - 18.

[4] Kulenovic, M. R. and M. K. Grammatikopoulos: Some comparison and oscilation results for first order differential equations and inequalities with a deviating arguments. J. Math. Anal. Appl. 131 (1988), $67-84$.

Received 14.06.2001; in revised form 07.01.2002 